\title{
Correction to: BFRIFP: Brain Functional Reorganization Inspired Filter Pruning
}

\author{
Shoumeng Qiu, Yuzhang Gu, and Xiaolin Zhang
}

\section{Correction to: \\ Chapter "BFRIFP: Brain Functional Reorganization Inspired Filter Pruning" in: I. Farkaš et al. (Eds.): Artificial Neural Networks and Machine Learning - ICANN 2021, LNCS 12894, https://doi.org/10.1007/978-3-030-86380-7_2}

The originally published article identified the incorrect corresponding author for the paper. The correct corresponding author has been updated now. 